Efficient preparation of macroporous poly(methyl methacrylate) materials from high internal phase emulsion templates

Khaled M. Althubeiti, Tommy S. Horozov

PII:

S1381-5148(19)30494-8

DOI: https://doi.org/10.1016/j.reactfunctpolym.2019.06.015

Reference: REACT 4307

To appear in: Reactive and Functional Polymers

Received date: 17 May 2019

Revised date:

18 June 2019

Accepted date:

20 June 2019" role="suppressed

Please cite this article as: K.M. Althubeiti and T.S. Horozov, Efficient preparation of macroporous poly(methyl methacrylate) materials from high internal phase emulsion templates, Reactive and Functional Polymers, https://doi.org/10.1016/ j.reactfunctpolym.2019.06.015

This is a PDF file of an unedited manuscript that has been accepted for publication. As a service to our customers we are providing this early version of the manuscript. The manuscript will undergo copyediting, typesetting, and review of the resulting proof before it is published in its final form. Please note that during the production process errors may be discovered which could affect the content, and all legal disclaimers that apply to the journal pertain.

(C)2019, Elsevier. This manuscript version is made available under the CC-BY-NC-ND 4.0 license http://creativecommons.org/licenses/by-nc-nd/4.0/ 


\title{
Efficient Preparation of Macroporous Poly(Methyl Methacrylate) Materials from High Internal Phase Emulsion Templates
}

Khaled M. Althubeiti ${ }^{1}$ and Tommy S. Horozov ${ }^{2, *}$ t.s.horozov@ hull.ac.uk

${ }^{1}$ Department of Chemistry - Faculty of Science, Taif University, Taif, 21974, P. O. Box 888, Saudi Arabia

${ }^{2}$ Department of Chemistry and Biochemistry, University of Hull, Cottingham Road, Hull, HU6 7RX, United Kingdom

\section{*Corresponding author.}

\begin{abstract}
Thermo- and photo-initiated polymerization of emulsion templates for making macroporous poly(methyl methacrylate) materials both suffer from low energy efficiency and require ted ious sample preparation (removal of inhibitors, inert atmosphere) or specialized equipment to be used. Here, an easy and efficient method for the preparation of such open pore materials with up to $93 \%$ porosity is demonstrated by using redox-initiated polymerization in the oil phase of high internal phase emulsion templates. The method eliminates the need of heating/irradiation and monomer purification, thus reducing the preparation costs without sacrificing the mechanical properties of the porous materials. It also allows of two component HIPE curing systems to be made, stored and used in the field for a rapid preparation of open pore monoliths in spaces with complex geometry. This is demonstrated by the in situ preparation of a filter and a static mixer for foam generation. The proposed method has a considerable potential for further practical exploitation in filtration, separation, microfluidics, chromatography and other fields where porous materials are used.
\end{abstract}

Keywords: (water-in-oil emulsion templates, redox-initiated polymerization, benzoyl peroxide, open pore polyHIPEs, polymer filters)

\section{Introduction}

The emulsion templating has been established as a versatile and reliable method for making macroporous polymeric materials with tunable porosity, pore sizes, connectivity $[1,2,3]$ and 
diverse applications in tissue engineering [1, 2, 4], gas storage [5], catalysis [6], chromatography [7], filtration and separation technology [8]. High internal phase emulsions (HIPEs) containing over 74 vol\% of the internal water phase dispersed as micrometer size droplets in the continuous oil phase of monomers have been commonly used for the preparation of polymeric materials with high porosity. The polymerization of the continuous phase of water-in-oil (w/o) emulsion templates gives polymerized HIPEs (polyHIPEs) which upon evaporation of the water droplets produce macroporous polymers. This approach has been successfully applied for the fabrication of polyHIPEs via thermo-initiated or photoinitiated polymerization of emulsions prepared from a variety of monomers and crosslinkers $[1,2,9]$. However, the polyHIPE preparation using methyl methacrylate (MMA) has been found challenging because the emulsion templates phase separated quickly upon heating [1]. Indeed, reports on the successful preparation of MMA-based polyHIPEs via thermo-initiated polymerization are rare $[10,11]$. There, purified monomers and inert atmosphere have been used to enhance the polymerization of w/o HIPE templates stabilized either by solid particles [10] or by $35 \%$ surfactant and up to $20 \%$ co-stabilizer in the oil phase [11]. Photo-initiated polymerization has been used with better success in the preparation of methacrylate-based polyHIPEs because it does not require heating [12]. However, its application is limited to thin transparent samples and requires powerful light sources. Both thermo- and photo-initiated polymerization approaches suffer from low energy efficiency and often require tedious sample preparation (removal of inhibitors, inert atmosphere, etc.) or specialized equipment to be used.

The use of redox-initiated polymerization as an alternative method has the advantage that it does not require an external energy source (heat of light) and the polymerization can occur at room temperature. Water-soluble redox couples, such as ammonium persulphate - N, N, N',N'-tetramethylethylenediamine (APS - TEMED), have been used for the preparation of polyHIPEs from hydrophilic or hydrophobic monomers $[13,14,15]$. The use of oil-soluble 
benzoyl peroxide (BPO) - tertiary amine redox couples for the bulk polymerization of MMA dates back to 1950s and has been widely utilized for rapid polymerization of acrylic-based materials in dentistry and medical restoration applications [16]. Recently, this approach was used for making injectable polyHIPE scaffolds by rapid polymerization of w/o HIPE templates prepared in inert atmosphere from purified dimethacrylates at elevated temperature $\left(37^{\circ} \mathrm{C}\right)$ [17]. In contrast, we focus our investigation on the preparation of porous PMMA materials, because the MMA is an inexpensive widely used monomer and the PMMA is a polymer with excellent mechanical properties, thus suitable for a variety of engineering applications [18, 19].

This work demonstrates for the first time that surfactant stabilized w/o HIPEs with a continuous oil phase of methyl methacrylate (MMA) and 10-30 vol\% ethylene glycol dimethacrylate (EGDMA) crosslinker can be easily polymerized via free radical initiation in the oil phase using a BPO - amine redox couple. The polymerization occurs at ambient conditions (room temperature, air) without the need of any monomer purification, heating or UV irradiation. The morphology and mechanical properties of the macroporous poly(methyl methacrylate) (PMMA) materials obtained by this method are compared to those produced by the traditional thermo-initiated polymerization using azobisisobutyronitrile (AIBN) initiator.

\section{Experimental}

\subsection{Materials}

Methyl methacrylate (MMA, $99 \%$ ), benzyl methacrylate (BeMA, $96 \%$ ), isobutyl methacrylate (97\%), ethylene glycol dimethacrylate (EGDMA, $98 \%$ ), benzoyl peroxide (BPO, $50 \%$ blend with dicyclohexyl phthalate as stabilizer), $N, N$-Dimethyl- $p$-toluidine (DMPT, $98.5 \%$ ), azobis isobutyronitrile (AIBN, $98 \%$ ), Pluronic ${ }^{\circledR}$ L-121 surfactant (average $\left.\mathrm{M}_{\mathrm{n}} \sim 4,400\right)$ and calcium chloride dihydrate $\left(\mathrm{CaCl}_{2} \cdot 2 \mathrm{H}_{2} \mathrm{O}, 99 \%\right)$ were purchased from SigmaAldrich

\subsection{Sample preparation}


All chemicals in redox-initiated polymerization experiments were used as received. Emulsion templates were prepared in volumes of $50 \mathrm{~mL}$ by adding deionized water dropwise (syringe pump, $4 \mathrm{~mL} \min ^{-1}$ ) to the oil phase (a mixture of MMA and 10-30 vol\% EGDMA) containing $1 \mathrm{w} / \mathrm{v} \%$ BPO initiator (as received) and $5 \mathrm{w} / \mathrm{v} \%$ Pluronic ${ }^{\circledR}$ L-121 surfactant in a $500 \mathrm{~mL}$ three-neck flask under continuous stirring with an overhead stirrer at $200 \mathrm{rpm}$. Once all the water phase was added, the emulsion was further stirred at $700 \mathrm{rpm}$ for a time equal to that spent for adding the water phase. The polymerization was triggered by adding the DMPT accelerator (12 $\mu \mathrm{L}$ per $1 \mathrm{~mL}$ oil phase) to the emulsion under continuous stirring for several minutes. Emulsion samples were left to polymerize in open cylindrical molds in air at room temperature overnight. The polyHIPE samples were purified by Soxhlet extraction with absolute ethanol for 24 hours, then dried in air at room temperature overnight. Alternatively, polyHIPEs were produced by mixing two emulsions, one containing $2 \mathrm{w} / \mathrm{v} \%$ BPO (as received) and the other $2.38 \mathrm{wt} / \mathrm{v} \%$ DMPT only, prepared in advance as described above. The polymerization was triggered by mixing equal volumes of both HIPEs.

The samples in thermo-initiated polymerization experiments were prepared as described above with the following modifications. The oil phase was prepared with $1.6 \mathrm{w} / \mathrm{v} \%$ AIBN (instead of BPO) from purified monomers passed three times through basic alumina to remove the inhibitors and purged with nitrogen before use. The water phase contained $0.12 \mathrm{M}$ $\mathrm{CaCl}_{2}$ and was degassed under vacuum for 10 minutes before the experiments. Nitrogen was flowing through the flask during the emulsification. The emulsion templates were polymerized in sealed molds inside a preheated to $70^{\circ} \mathrm{C}$ oven overnight.

\subsection{Characterization}

The pot life of redox-initiated HIPE templates was determined by measuring the apparent viscosity of the polymerizing samples using a home-built setup (Figure S1, S2 and S3). The porosity was calculated by Equation S1 from the density and volume of the porous samples, and the polymer density. Pore structures were studied by scanning electron microscopy (SEM 
EVO 60, Carl Zeiss) of polyHIPE samples coated with $1 \mathrm{~nm}$ thick gold layer by using a sputter coater. The sizes of 400 pores and pore throats were measured from the SEM images using Image $\mathbf{J}$ software. The median pore throat diameters were determined from their size distribution. The true median pore diameters were determined by stereological analysis following the Saltykov method [20]. The mechanical tests were done with a Mark-10 ESM303 instrument $\left(2.5 \mathrm{kN}\right.$ load cell) at a compression rate of $1.2 \mathrm{~mm} \mathrm{~min}^{-1}$ at room temperature (see Supporting information).

\section{Results and discussion}

Examples of typical w/o HIPE samples (M75R - M90R) prepared in our study are shown in Table 1. Stable w/o emulsion templates with $75-90$ vol\% internal water phase were made by stirring deionized water and the oil phase (a mixture of MMA and EGDMA) containing 1 $\mathrm{w} / \mathrm{v} \%$ BPO initiator (as received) and $5 \mathrm{w} / \mathrm{v} \%$ Pluronic $^{\circledR}$ L-121 surfactant as an emulsifier. The polymerization was triggered by adding $\mathrm{N}, \mathrm{N}$-dimethyl-p-toluidine (DMPT) accelerator to the stirred emulsion template and conducted in open molds in air at room temperature. We have found that all samples solidified to polyHIPE monoliths without visible defects (Figure 1a-d, insets) within 30 - 35 minutes after adding the accelerator. The maximum temperature reached during the polymerization has monotonically decreased from $35^{\circ} \mathrm{C}$, at 75 vol\%, to 26 ${ }^{\circ} \mathrm{C}$ at 90 vol\% water phase. This is because the smaller amount of polymerizing monomers produces less heat which is absorbed by the larger amount of water in the more concentrated emulsions. The lower temperature during the polymerization of the HIPEs with larger water content has slowed down their curing rate and prolonged the pot life.

As expected, the porosity of purified dry polyHIPE samples has increased with the water content of the HIPE templates and is in the range $79-93 \%$ (Table 1). The SEM images shown in Figure 1 (a) - (d) reveal that the porous materials have an open pore structure of nearly spherical pores connected by circular pore throats. The SEM image analysis has shown that the pores and pore throats have relatively broad size distributions (Figure S4, Table S1 
and S2). The median pore diameter increases with the increase of the water volume fraction in the emulsion template, thus suggesting that the size of the template droplets has been bigger in the more concentrated emulsions. This has been supported by optical microscopy observations of the HIPE templates which showed up to $70 \%$ increase of the median droplet diameter when the water volume fraction was increased from 75 to 90 vol\%. This seems reasonable because the surfactant concentration in the oil phase has been kept constant, hence the total amount of surfactant progressively decreases with the increase of water content. The median pore throat diameter follows the trend observed for the pore size, but its increase is even more significant.

The mechanical tests on purified polyHIPE samples have revealed that the Young modulus and compressive strength decrease with the porosity, as expected $[18,21,22]$. However, Young's moduli of the samples with porosity 88 and $93 \%$ are very close to the estimated expected moduli, $E_{f}$, (Table 1) and 50 - 100 times larger than the values reported for PMMA polyHIPEs produced by thermo-initiated polymerization of emulsions with 86 vol\% water phase [11]. For better comparison, we have tried to polymerize HIPE templates with the same composition, but using the BPO as a thermo-initiator at $70-80{ }^{\circ} \mathrm{C}$, i.e. without adding DMPT to the emulsions. All attempts have failed because the emulsion templates phase separated when heated. We have obtained better results by replacing the BPO with the more efficient and widely used AIBN thermo-initiator in the oil phase. However, the thermo-initiated polymerization of HIPE templates has turned out to be very challenging. We had to purify the monomers by removing the inhibitors, degas and add $0.12 \mathrm{M} \mathrm{CaCl}_{2}$ to the aqueous phase, use nitrogen atmosphere throughout the HIPE preparation and polymerize them in sealed molds at $70{ }^{\circ} \mathrm{C}$ in an oven for several hours. Nevertheless, the w/o HIPE templates with 85 and 90 vol\% inner aqueous phase destabilized during the heating and quality polyHIPEs were not produced. The results for samples M75T and M80T with 75 and 80 vol\% aqueous content, respectively, are summarized in Table 1 and SEM images of the polyHIPEs are shown in 
Figure 1 (e) and (f). In both cases, the polyHIPEs have an open pore structure similar to that of samples produced by redox-initiated polymerization (Figure 1a and b). They have slightly higher porosities but similar pore and pore throat diameters to the respective M75R and M80R samples. The compressive strength of thermo-initiated polyHIPEs is comparable to that of the redox-initiated materials with similar porosity, however, their Young modulus is almost two times lower (e.g. 26 vs $46 \mathrm{MPa}$ at $88 \%$ porosity) and significantly smaller than the estimated expected modulus, $E_{f}$, (Table 1 ). The polyHIPE materials produced by thermoinitiated polymerization were more brittle and crumbled during the compressive tests (cf. insets in Figure 1). This behavior correlates with the observation that the pore walls of polyHIPE samples produced by thermo-initiation have a grainy structure in contrast to the smooth polymer walls in redox-initiated samples (Figure 2). The grainy polymer structure could be due to the fact that the polymerization at $70{ }^{\circ} \mathrm{C}$ has occurred faster in a less controllable way in contrast to the redox-initiated polymerization in which the temperature did not exceed $35{ }^{\circ} \mathrm{C}$. The increased water solubility in the oil phase at an elevated temperature could also play a role. The effect of these factors on the polymer microstructure needs further investigation.

Our results demonstrate that the redox-initiation method has significant advantages over the traditional methods of making MMA-based porous materials by thermo- or photo-initiated polymerization of HIPE templates: (i) it considerably simplifies the preparation by eliminating the monomer purification, the use of inert atmosphere and electrolyte in the aqueous emulsion phase; (ii) macroporous polymers with open pore structure and improved mechanical properties are produced without the need of an external source of heating or irradiation. Moreover, we have found that this method also works well when the MMA is replaced by other methacrylate, such as benzyl methacrylate (BeMA) or isobutyl methacrylate, and produces polyHIPEs with morphology similar to the macroporous PMMA materials (Figure 3 and S5). The use of an initiator-accelerator redox couple offers additional 
advantages for practical applications by allowing the preparation of two component curing systems which must be mixed to trigger the polymerization [16, 17]. Following this approach, we have formulated two separate HIPE templates: one containing the BPO initiator and the other the DMPT accelerator only. These emulsions can be stored, mixed just before use, then delivered and polymerized in a variety of moulds and spaces with complex geometry to give open pore monoliths ready to be used as porous media shortly after the start of polymerisation. For example, we have used two BeMA-based HIPEs (80 vol\% water) for the in situ preparation of a filter and a static mixer for foam generation, both functional in less than 20 minutes after mixing the emulsions (Figure 3 and Video S1). The two component MMAbased system gave similar results but the filter preparation took 15 min longer due to slower polymerization (Video S2). However, its curing time was significantly reduced by increasing the crosslinker concentration without compromising the mechanical properties of the polyHIPE (Table S3). The long-term stability of those emulsions was considerably improved by adding electrolyte to the aqueous phase (Figure S6) and they were able to polymerize even at $10{ }^{\circ} \mathrm{C}$ (although for a two times longer time), thus showing a potential for their use in the field.

\section{Conclusions}

In summary, we demonstrate an efficient method for the preparation of open pore polyHIPE materials by using BPO - DMPT redox-initiated polymerization of w/o HIPE templates formulated with MMA or other methacrylates. The method eliminates the need of heating/irradiation and monomer purification, thus reducing the costs without sacrificing the mechanical properties of the porous materials. It also allows of two component HIPE curing systems to be made, stored and used in the field for a rapid preparation of open pore monoliths in spaces with complex geometry. Therefore, the proposed method has a significant potential for further practical exploitation in filtration, separation technology, microfluidics, chromatography and other fields utilizing porous materials. 


\section{Supporting Information}

Supporting Information is available from the Journal or from the author.

\section{Acknowledgements}

K. M. Althubeiti is grateful to Taif University and the Saudi cultural bureau, London for the financial support of his Ph.D. studies at the University of Hull.

\section{Data availability}

The raw/processed data required to reproduce these findings cannot be shared at this time due to technical and time limitations. Data will be made available on request.

\section{References}

[1] N. R. Cameron, Polymer 2005, 46, 1439.

[2] M. S. Silverstein, Polymer 2014, 55, 304.

[3] a) V. O. Ikem, A. Menner, A. Bismarck, Angew. Chem. Int. Ed. 2008, 47, 8277; b) V.

O. Ikem, A. Menner, T. S. Horozov, A. Bismarck, Adv. Mater. 2010, 22, 3588.

[4] a) S. Caldwell, D. W. Johnson, M. P. Didsbury, B. A. Murray, J. J. Wu, S. A.

Przyborski, N. R. Cameron, Soft Matter 2012, 8, 10344; b) J. Naranda, M. Sušec, U. Maver, L. Gradišnik, M. Gorenjak, A. Vukasović, A. Ivković, M. S. Rupnik, M. Vogrin, P. Krajnc, Sci. Rep. 2016, 6, 28695.

[5] F. Su, C. L. Bray, B. Tan, A. I. Cooper, Adv. Mater. 2008, 20, 2663.

[6] a) F. Fernández-Trillo, J. C. M. van Hest, J. C. Thies, T. Michon, R. Weberskirch, N. R. Cameron, Adv. Mater. 2009, 21, 55; b) I. Pulko, J. Wall, P. Krajnc, N. R. Cameron, Chem. Eur. J. 2010, 16, 2350; c) C. E. Chan-Thaw, A. Villa, P. Katekomol, D. Su, A. Thomas, L. Prati, Nano Lett. 2010, 10, 537. 
[7] P. Krajnc, N. Leber, D. Štefanec, S. Kontrec, A. Podgornik, J. Chromatogr. A 2005, $1065,69$.

[8] a) Z. Bhumgara, Filtr. Sep. 1995, 32, 245; b) M. Tebboth, A. Menner, A. Kogelbauer,

A. Bismarck, Curr. Opin. Chem. Eng. 2014, 4, 114.

[9] Q. Jiang, A. Menner, A. Bismarck, Polymer 2016, 97, 598.

[10] S. Zhang, J. Chen, Chem. Commun. 2009, 2217.

[11] Z. Li, H. Liu, L. Zeng, H. Liu, Y. Wang, J. Mater. Sci. 2016, 51, 9005.

[12] a) S. D. Kimmins, P. Wyman, N. R. Cameron, React. Funct. Polym. 2012, 72, 947; b)

D. W. Johnson, C. Sherborne, M. P. Didsbury, C. Pateman, N. R. Cameron, F. Claeyssens, Adv. Mater. 2013, 25, 3178; c) S. Huš, P. Krajnc, Polymer 2014, 55, 4420.

[13] S. Kovačič, D. Štefanec, P. Krajnc, Macromolecules 2007, 40, 8056.

[14] S. Kovačič, M. S. Silverstein, Macromol. Rapid Commun. 2016, 37, 1814.

[15] S. Kovačič, N. Drašinac, A. Pintar, E. Žagar, Langmuir 2018, 34, 10353.

[16] a) P. K. Vallittu, I. E. Ruyter, S. Buykuilmaz, Eur. J. Oral. Sci. 1998, 106, 588; b) R. Vaishya, M. Chauhan, A. Vaish, J. Clin. Orthop. Trauma. 2013, 4, 157.

[17] R. S. Moglia, M. Whitely, P. Dhavalikar, J. Robinson, H. Pearce, M. Brooks, M. Stuebben, N. Cordner, E. Cosgriff-Hernandez, Biomacromolecules 2014, 15, 2870.

[18] L. J. Gibson, M. F. Ashby, Cellular Solids Structure and Properties, 2nd ed., Cambridge University Press, Cambridge, UK 1997.

[19] Polymer handbook, 4th ed. (Eds: J. Brandrup, E.H. Immergut, E.A. Grulke) Wiley, New York, USA 1999.

[20] a) S. A. Saltykov, Stereometric metallography, Metallurgy, Moscow, USSR 1976; b) D. L. Sahagian, A. A. Proussevitch, J. Volcanol. Geotherm. Res. 1998, 84, 173.

[21] G. Ceglia, A. Merlin, P. Viot, V. Schmitt, O. Mondain-Monval, J. Porous Mater. 2014, $21,903$.

[22] S. Kovačič, E. Žagar, C. Slugovc, Polymer 2019, 169, 58 


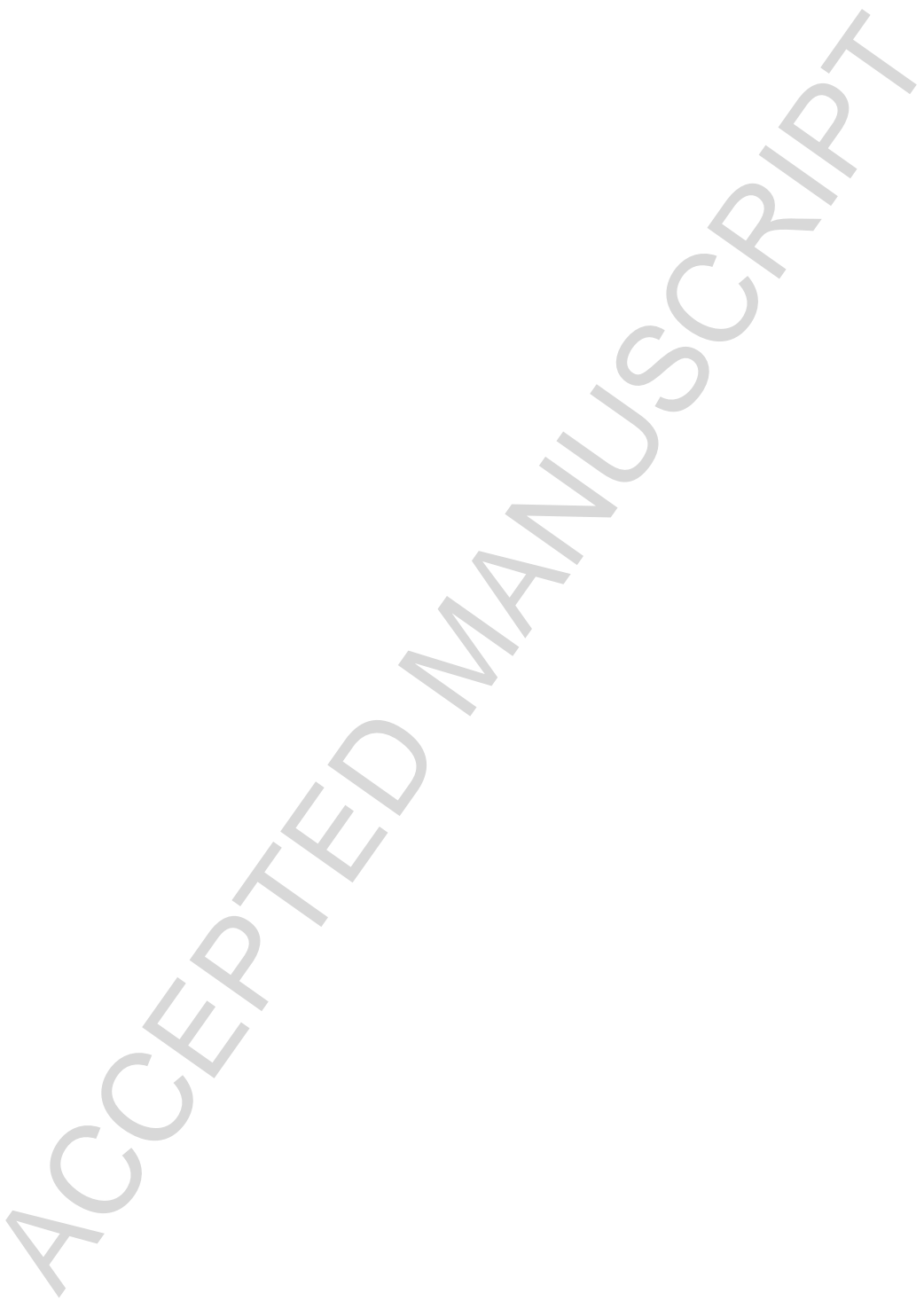


Table 1. Internal phase volume fraction $\left(\phi_{\mathrm{w}}\right)$, curing conditions and pot life $\left(t_{\mathrm{p}}\right)$ of w/o HIPE templates, and porosity $(\mathrm{P})$, median pore diameter $\left(\mathrm{d}_{\mathrm{p}}\right)$, median pore throat diameter $\left(\mathrm{d}_{\mathrm{t}}\right)$, compressive strength $(\sigma)$, Young's modulus $(\mathrm{E})$ and the ratio of measured to expected Young's modulus $\left(\mathrm{E} / \mathrm{E}_{\mathrm{f}}\right)$ of the respective polyHIPEs produced by redox-initiated $(\mathrm{M} 75 \mathrm{R}-$ M90R) or thermo-initiated (M75T and M80T) polymerization. The continuous oil phase of emulsion templates contains 85 vol\% MMA, 15 vol\% EGDMA, 5 w/v\% Pluronic ${ }^{\circledR}$ L-121 surfactant and redox- or thermo-initiator.

\begin{tabular}{|l|c|c|c|c|c|c|c|c|c|}
\hline Sample & $\phi_{\mathrm{w}}[\mathrm{vol} \%]$ & Curing & $\mathrm{t}_{\mathrm{p}}[\mathrm{min}]^{\mathrm{d})}$ & $\mathrm{P}[\%]$ & $\mathrm{d}_{\mathrm{p}}[\mu \mathrm{m}]$ & $\mathrm{d}_{\mathrm{t}}[\mu \mathrm{m}]$ & $\sigma[\mathrm{MPa}]$ & $\mathrm{E}[\mathrm{MPa}]$ & $\mathrm{E} / \mathrm{E}_{f}^{\mathrm{e}}$ \\
\hline M75R $^{\mathrm{a})}$ & 75 & room temp. $^{\mathrm{c}}$ & $16 \pm 1$ & $79 \pm 2$ & $16 \pm 1$ & $2.4 \pm 0.2$ & $5.1 \pm 0.1$ & $85 \pm 1$ & 0.6 \\
\hline M80R $^{\mathrm{a})}$ & 80 & room temp. $^{\mathrm{c}}$ & $18 \pm 1$ & $84 \pm 2$ & $23 \pm 1$ & $3.7 \pm 0.4$ & $3.2 \pm 0.1$ & $66 \pm 1$ & 0.8 \\
\hline M85R $^{\mathrm{a})}$ & 85 & room temp. $^{\mathrm{c}}$ & $22 \pm 1$ & $88 \pm 2$ & $26 \pm 1$ & $4.5 \pm 0.5$ & $1.8 \pm 0.1$ & $46 \pm 1$ & 1.0 \\
\hline M90R $^{\mathrm{a})}$ & 90 & room temp. $^{\mathrm{c}}$ & $23 \pm 1$ & $93 \pm 2$ & $29 \pm 2$ & $6.4 \pm 0.6$ & $0.6 \pm 0.1$ & $14 \pm 1$ & 0.9 \\
\hline M75T $^{\mathrm{b})}$ & 75 & oven, $70^{\circ} \mathrm{C}$ & - & $82 \pm 2$ & $17 \pm 1$ & $2.4 \pm 0.2$ & $3.5 \pm 0.4$ & $41 \pm 9$ & 0.4 \\
\hline M80T $^{\mathrm{b})}$ & 80 & oven, $70^{\circ} \mathrm{C}$ & - & $88 \pm 2$ & $17 \pm 1$ & $2.7 \pm 0.2$ & $1.7 \pm 0.1$ & $26 \pm 4$ & 0.6 \\
\hline
\end{tabular}

${ }^{a}$ The oil phase contains $1 \mathrm{w} / \mathrm{v} \%$ BPO initiator (as received) and 1.19 vol\% DMPT accelerator, the water phase is deionized water; ${ }^{b}$ The oil phase contains $1.6 \mathrm{w} / \mathrm{v} \%$ AIBN thermo-initiator, the water phase is a degassed $0.12 \mathrm{M} \mathrm{CaCl}_{2}$ solution; ${ }^{\mathrm{c})}$ Open molds in air at $23 \pm 2{ }^{\circ} \mathrm{C}$; ${ }^{\mathrm{d})} \mathrm{Time}$ taken for doubling the initial apparent viscosity; ${ }^{\mathrm{e})}$ The expected Young modulus $\left(E_{f}\right)$ is calculated by ${ }^{[15]} E_{f}=E_{p}\left(\rho_{f} / \rho_{p}\right)^{2}$, where $\rho_{f}$ is the measured porous polymer density, $\rho_{p}=$ $1200 \mathrm{~kg} \mathrm{~m}^{-3}$ and $E_{p}=3100 \mathrm{MPa}$ are the density and Young modulus of PMMA, respectively. ${ }^{[16]}$ 

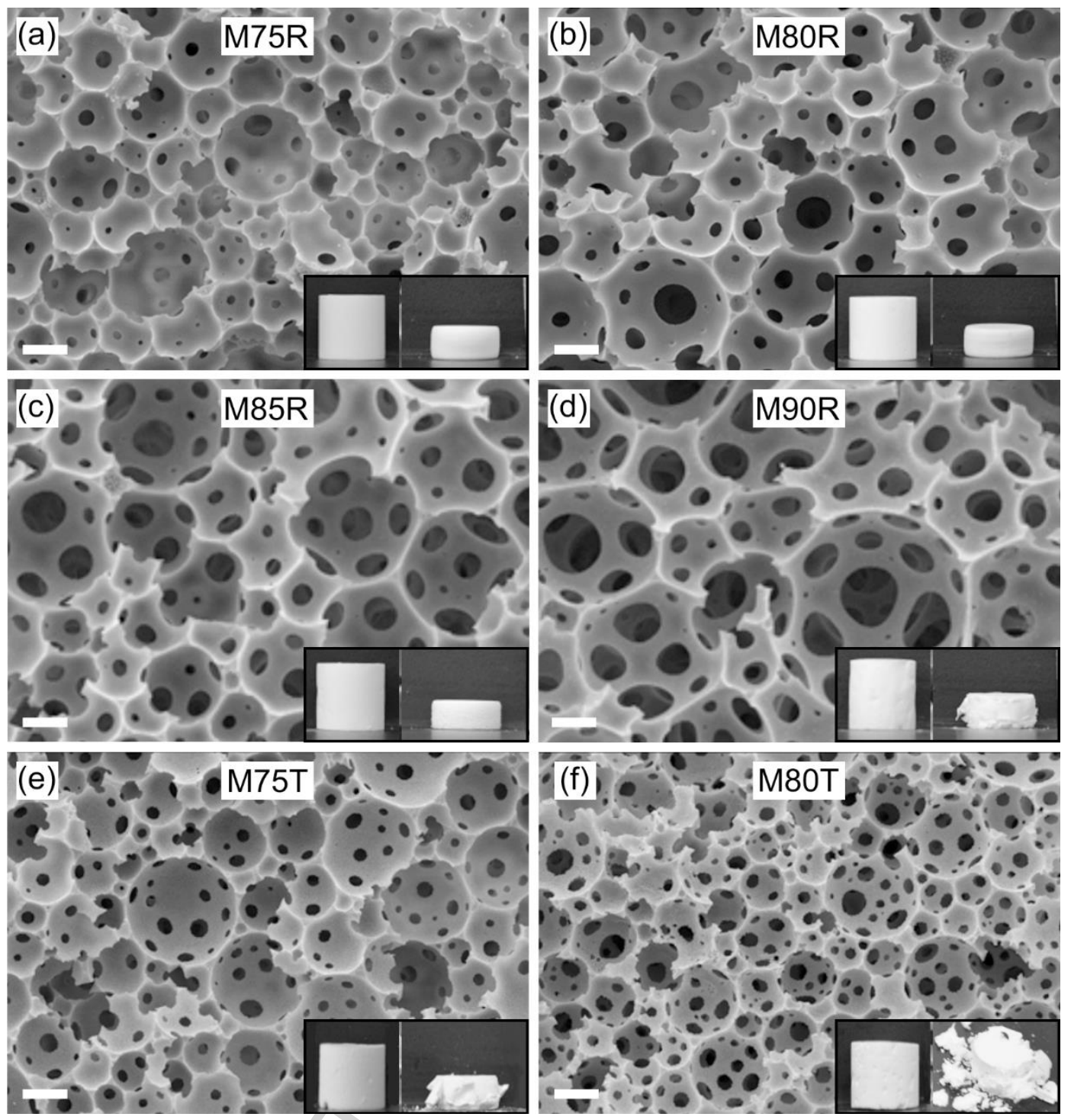

Figure 1. SEM images of polyHIPEs produced by: $(a-d)$ redox-initiated and $(e, f)$ thermoinitiated polymerization (see Table 1). Scale bars are $10 \mu \mathrm{m}$. Insets show digital camera images of the respective cylindrical samples $(10.3 \mathrm{~mm}$ in diameter) taken before (left) and after (right) the compression test.

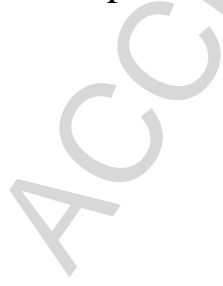



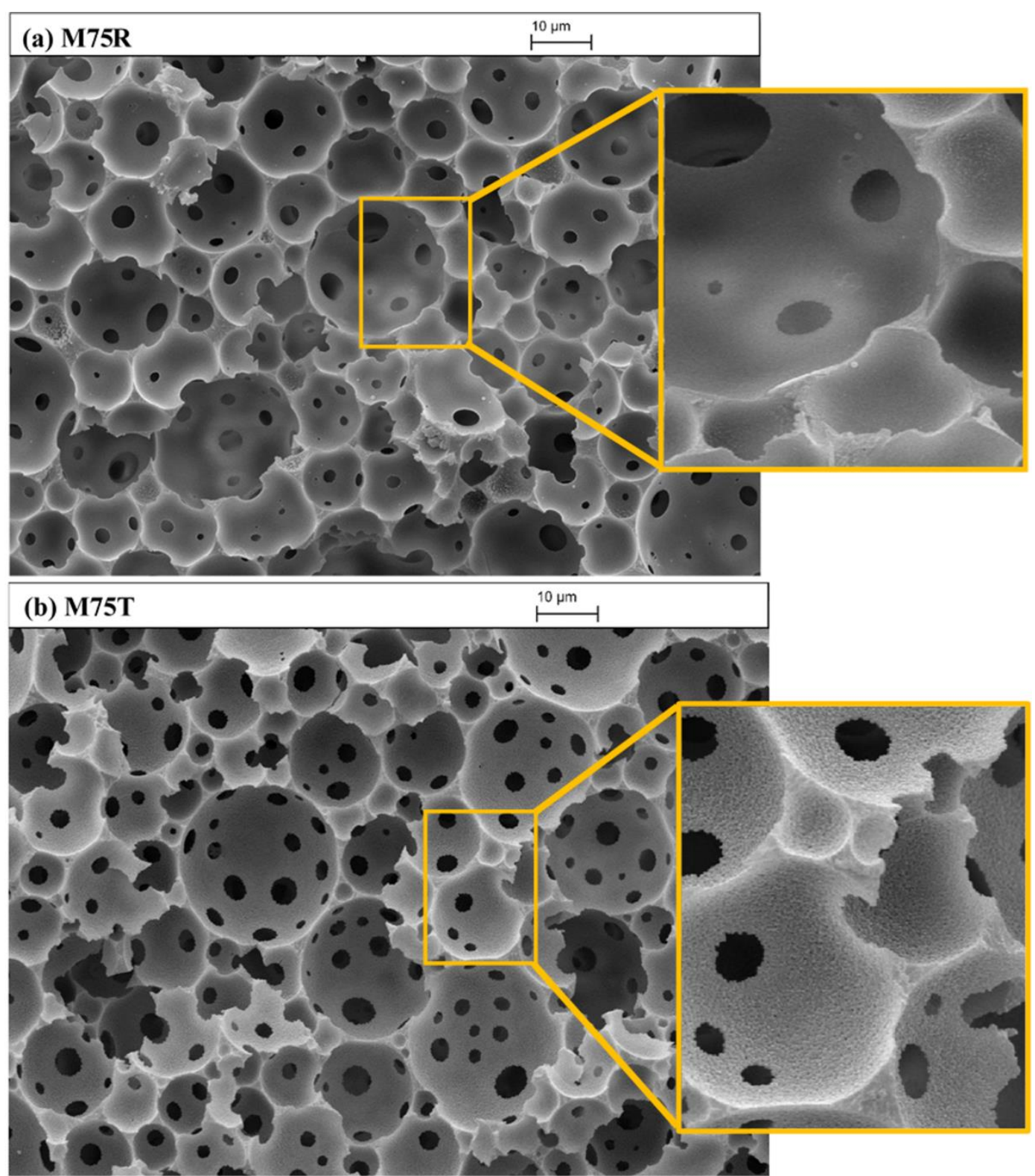

Figure 2. SEM images of polyHIPE samples produced from w/o emulsion templates by (a) redox-initiated and (b) thermo-initiated polymerization (see Table 1). The zoomed areas clearly show that the polymer surface in (a) is smooth while that in (b) has a grainy structure.

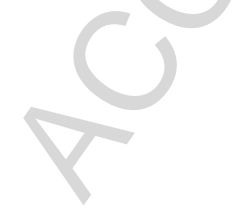



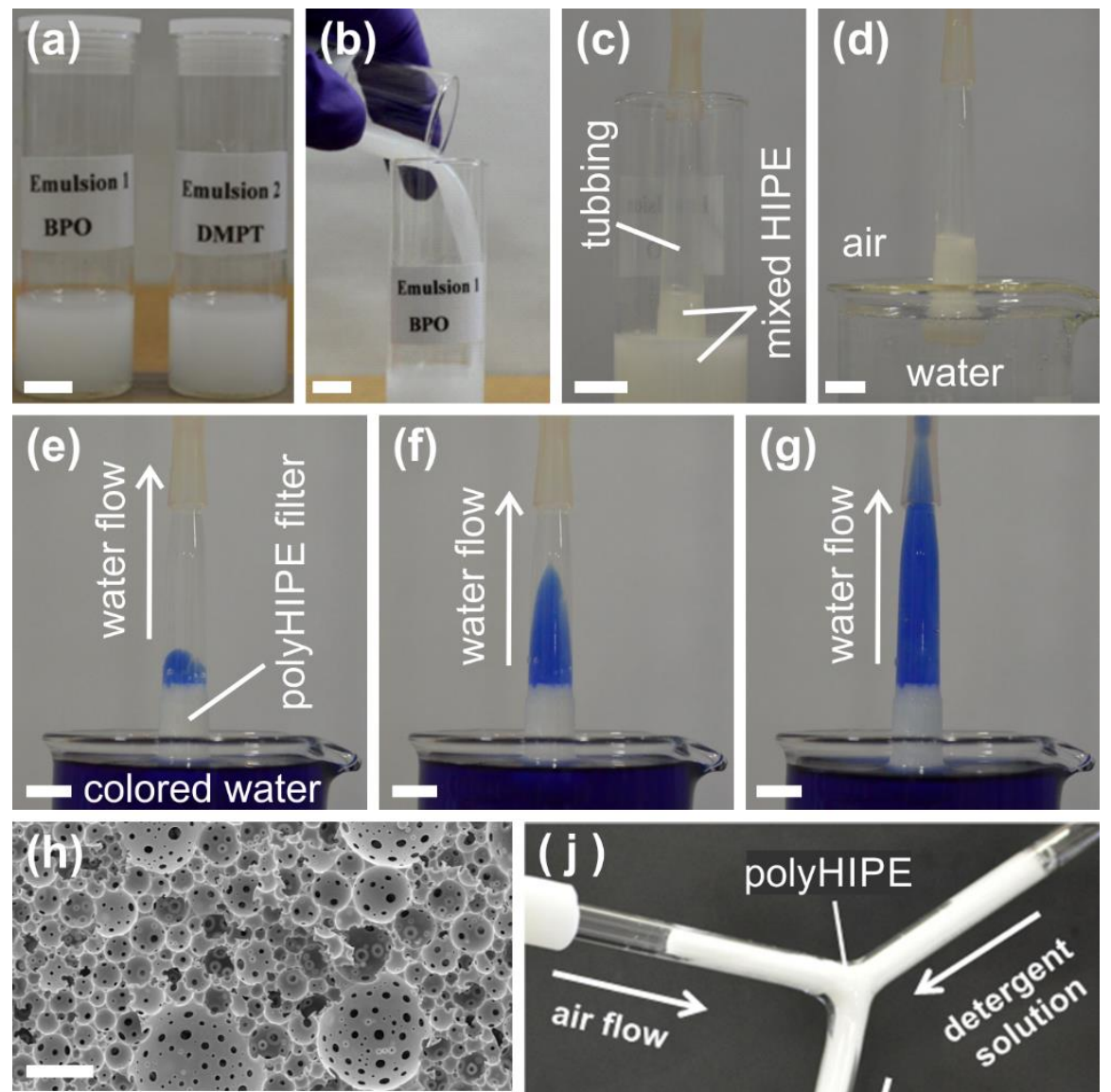

\section{polyHIPE}
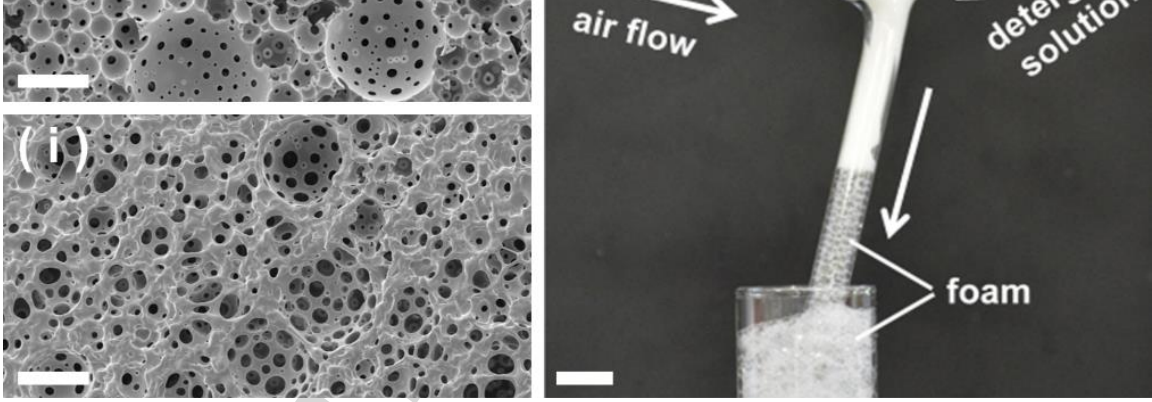

Figure 3. $(\mathrm{a}-\mathrm{g})$ In situ preparation of a polyHIPE filter: $(\mathrm{a}-\mathrm{b})$ mixing of two w/o HIPEs with 20 vol\% oil phase (BeMA:EGDMA $=85: 15 \mathrm{v} / \mathrm{v})$ containing $5 \mathrm{w} / \mathrm{v} \%$ Pluronic ${ }^{\circledR} \mathrm{L}-121$ and $2 \mathrm{w} / \mathrm{v} \%$ BPO (as received, emulsion 1) or 2.38 vol\% DMPT (emulsion 2); (c) a plug of the mixed HIPE is sucked in a plastic tubing prefilled with water; (d) the plug is left to polymerize immersed in water for $15 \mathrm{~min}$; $(\mathrm{e}-\mathrm{f})$ colored water is sucked through the polymerized HIPE plug showing that the polyHIPE is permeable to water; (h, i) SEM images taken (h) inside and (i) at the bottom surface of the polyHIPE confirming its open pore structure. (j) polyHIPE produced in a similar way inside a ' $\mathrm{Y}$ '-shaped glass tubbing is used as a static mixer for foam generation. Scale bars are $(a-g, j)-10 \mathrm{~mm}$; (h, i) - $100 \mu \mathrm{m}$. For more details see the Supporting information. 


\section{Figure captions}

Figure 1. SEM images of polyHIPEs produced by: $(a-d)$ redox-initiated and $(e, f)$ thermoinitiated polymerization (see Table 1). Scale bars are $10 \mu \mathrm{m}$. Insets show digital camera images of the respective cylindrical samples $(10.3 \mathrm{~mm}$ in diameter) taken before (left) and after (right) the compression test.

Figure 2. SEM images of polyHIPE samples produced from w/o emulsion templates by (a) redox-initiated and (b) thermo-initiated polymerization (see Table 1). The zoomed areas clearly show that the polymer surface in (a) is smooth while that in (b) has a grainy structure.

Figure 3. $(\mathrm{a}-\mathrm{g})$ In situ preparation of a polyHIPE filter: $(\mathrm{a}-\mathrm{b})$ mixing of two w/o HIPEs with 20 vol\% oil phase (BeMA:EGDMA = 85:15 v/v) containing $5 \mathrm{w} / \mathrm{v} \%$ Pluronic ${ }^{\circledR}$ L-121 and $2 \mathrm{w} / \mathrm{v} \%$ BPO (as received, emulsion 1) or 2.38 vol\% DMPT (emulsion 2); (c) a plug of the mixed HIPE is sucked in a plastic tubing prefilled with water; (d) the plug is left to polymerize immersed in water for $15 \mathrm{~min}$; ( $\mathrm{e}-\mathrm{f}$ ) colored water is sucked through the polymerized HIPE plug showing that the polyHIPE is permeable to water; (h, i) SEM images taken (h) inside and (i) at the bottom surface of the polyHIPE confirming its open pore structure. (j) polyHIPE produced in a similar way inside a ' $\mathrm{Y}$ ' -shaped glass tubbing is used as a static mixer for foam generation. Scale bars are $(\mathrm{a}-\mathrm{g}, \mathrm{j})-10 \mathrm{~mm}$; $(\mathrm{h}, \mathrm{i})-100 \mu \mathrm{m}$. For more details see the Supporting information. 